Research Article

\title{
On Locating-Dominating Set of Regular Graphs
}

\author{
Anuwar Kadir Abdul Gafur $\mathbb{D D}^{1}$ and Suhadi Wido Saputro $\mathbb{D}^{2}$ \\ ${ }^{1}$ Department of Mathematics, Pasific Morotai University, Morotai, Indonesia \\ ${ }^{2}$ Department of Mathematics, Bandung Institute of Technology, Bandung, Indonesia \\ Correspondence should be addressed to Suhadi Wido Saputro; suhadi@math.itb.ac.id
}

Received 16 July 2021; Accepted 28 August 2021; Published 25 September 2021

Academic Editor: Muhammad Kamran Siddiqui

Copyright (c) 2021 Anuwar Kadir Abdul Gafur and Suhadi Wido Saputro. This is an open access article distributed under the Creative Commons Attribution License, which permits unrestricted use, distribution, and reproduction in any medium, provided the original work is properly cited.

\begin{abstract}
Let $G$ be a simple, connected, and finite graph. For every vertex $v \in V(G)$, we denote by $N_{G}(v)$ the set of neighbours of $v$ in $G$. The locating-dominating number of a graph $G$ is defined as the minimum cardinality of $W \subseteq V(G)$ such that every two distinct vertices $u, v \in V(G) \backslash W$ satisfies $\varnothing \neq N_{G}(u) \cap W \neq N_{G}(v) \cap W \neq \varnothing$. A graph $G$ is called $k$-regular graph if every vertex of $G$ is adjacent to $k$ other vertices of $G$. In this paper, we determine the locating-dominating number of $k$-regular graph of order $n$, where $k=n-2$ or $k=n-3$
\end{abstract}

\section{Introduction}

Given a simple, connected, and finite graph $G$. The neighbours of a vertex $v$ of $G$ are defined as the vertex set $N_{G}(v)=\{u \in V(G) \mid u v \in E(G)\}$. A set of vertices $W \subseteq V(G)$ is called a locating-dominating set of a graph $G$ if every two distinct vertices $u, v \in V(G) \backslash W$ satisfies $\varnothing \neq N_{G}(u) \cap$ $W \neq N_{G}(v) \cap W \neq \varnothing$. The minimum cardinality of locatingdominating sets of $G$ is called the locating-dominating number of $G$, denoted by $\lambda(G)$. This concept was introduced by Slater $[1,2]$.

Let us model a building floor as a graph. Locatingdominating set can be used to determine an exact location of a fire alarm which sends a signal when detecting a fire in any of its adjacent vertices. The activated signals will univocally determine the place of the fire.

Charon et al. [3] have proved that determining locatingdominating number of a graph is NP-complete problem which is reduced from 3-SAT. However, some results for certain classes of graphs have been obtained, such as paths [2], cycles [4], stars [5], complete graphs [6], bipartite graphs $[7,8]$, complete multipartite graphs [5], wheels [7], twin-free graphs $[9,10]$, and hypergraphs [11]. In [12], Balbuena et al. investigated a locating-dominating set of graphs with girth at least 5. In the other hand, Rajasekar and Nagarajan [13] studied the locating-dominating number of a graph containing a bridge.

Furthermore, some authors have been characterized all graphs with a given locating-dominating number. Henning and Oellermann [6] have proved that for a connected graph $G$ of order $n \geq 2, \lambda(G)=n-1$ if and only if $G$ is either complete graphs $K_{n}$ or star graphs $K_{1, n-1}$. They also characterized all graphs $G$ of order $n \geq 4$ with locating-dominating number $n-2$. Meanwhile, Caceres et al. [7] have proved that there are 16 nonisomorphic graphs $G$ satisfying $\lambda(G)=2$.

Some authors also have determined the locating-dominating number of graphs obtained from a product graphs. Canoy and Malacas [14] provided the lower and upper bounds for the locating-dominating number of corona product graphs. They also determined an exact value of the locating-dominating number of a composition of graphs between $G$ and $H$ where $G$ is a connected totally point determining graph and $H$ is a nontrivial connected graph. An exact value of the locating-dominating number of comb product of any two connected graphs of order at least two has been determined by Pribadi and Saputro [15]. Murtaza et al. [16] studied the locating-domination number of functigraphs of complete graphs. A study of a locatingdominating set of a graph by adding a universal vertex can be seen in [17]. 
In this paper, we consider a regular graph. A graph $G$ is called $k$-regular graph if every vertex in $G$ is adjacent to $k$ other vertices. Since every vertex of $G$ is adjacent to the same number of vertices of $G$, every vertex of $G$ has the same probability to distinguish some distinct vertices of $G$. Let $G=(V, E)$ be a model of the multiprocessor system, such that $V(G)$ is the set of processors and $E(G)$ is the set of links between processors. Assume that at most one processor is malfunctioning and we want to test the system and find the faulty processor. Some processors can be chosen and assigned to check their neighbours. In case a selected processor detects a fault, it sends an alarm signal. Since we need an exact location of a faulty processor, we must choose some processors such that the chosen processors can uniquely tell the location of the malfunctioning processor. Then, a locating-dominating set of $G$ can be used to choose those processors.

Bertrand et al. [4] have initiated the study of the locatingdominating number on regular graph. They determined the locating-dominating number of 2-regular connected graphs (cycles). The locating-dominating number of $(n-1)$-regular graph of order $n$ can be seen in [7]. In this paper, we determine the locating-dominating number of $k$-regular graph of order $n$ where $k=n-2$ or $k=n-3$.

The purpose of this paper is to further investigate the locating-dominating number of certain family of graphs, namely, to determine the locating-dominating number of certain regular graphs. We obtain two main results, one of them is the following result related with an $(n-2)$-regular graph.

Theorem 1. For $n \geq 4$, let $G$ be an $(n-2)$-regular graph. Then, $\lambda(G)=n / 2$.

Our second result is related with an $(n-3)$-regular graph. In preparing the proof for the second result, we are able to obtain the intermediate result as follows.

Theorem 2. For $m \geq 7$, let $H=K_{m} \backslash E\left(C_{m}\right)$. Then, $\lambda(H)=\lceil(2 m-2) / 5\rceil$.

For $n \geq 5$, we consider certain cycles contained in a complete graph $K_{n}$. In this paper, we assume that a cycle contains at least three vertices. For $r \in\{1,2, \ldots,\lfloor n / 3\rfloor\}$, let $R_{1}, R_{2}, \ldots, R_{r}$ be $r$ disjoint cycles contained in $K_{n}$ such that $V\left(R_{1}\right) \cup V\left(R_{2}\right) \cup \cdots \cup V\left(R_{r}\right)=V\left(K_{n}\right)$. Note that an $(n-3)$-regular graph is isomorphic to $K_{n}\left(E\left(R_{1}\right) \cup\right.$ $\left.E\left(R_{2}\right) \cup \cdots \cup E\left(R_{r}\right)\right)$. In case $r=1$, the locating-dominating number of an $(n-3)$-regular graph of order at least 7 has been determined in Theorem 2. In Theorem 3, we provide the locating-dominating number of an $(n-3)$-regular graph of order $n \geq 5$ with $1 \leq r \leq\lfloor n / 3\rfloor$.

Theorem 3. For $n \geq 5$ and $1 \leq r \leq\lfloor n / 3\rfloor$, let $R_{1}, R_{2}, \ldots, R_{r}$ be $r$ disjoint cycles contained in $K_{n}$ such that $V\left(R_{1}\right) \cup V\left(R_{2}\right) \cup \cdots \cup V\left(R_{r}\right)=V\left(K_{n}\right)$. For $i \in\{1,2, \ldots, r\}$, let $G=K_{n} \backslash\left(E\left(R_{1}\right) \cup E\left(R_{2}\right) \cup \cdots \cup E\left(R_{r}\right)\right), \quad m_{i}=\left|V\left(R_{i}\right)\right|$, and $G_{i}=K_{m_{i}} \backslash E\left(R_{i}\right)$. If $k$ is the number of cycles $R_{i}$ of order $m_{i} \geq 7$ and $m_{i} \equiv 1$ or $3(\bmod 5)$, then

$$
\lambda(G)= \begin{cases}\sum_{i=1}^{r} \lambda\left(G_{i}\right), & \text { if } k \leq 1, \\ k-1+\sum_{i=1}^{r} \lambda\left(G_{i}\right), & \text { if } k \geq 2 .\end{cases}
$$

\section{2. $(n-2)$-Regular Graph and Proof of Theorem 1}

Theorem 1 is a direct consequence of Lemmas 1 and 2 in this section.

In this section, we define $G$ as an $(n-2)$-regular graph of order $n \geq 4$. Note that if we count the sum of degree of all vertices, then every edge will be counted twice. Therefore, we have $2|E(G)|=n(n-2)$. It implies that $n$ must be even.

Now, we can define $V(G)=\left\{x_{i}, y_{i} \mid 1 \leq i \leq(n / 2)\right\}$ and $E(G)=\{u v \mid u, v \in V(G)\} \backslash\left\{x_{i} y_{i} \mid 1 \leq i \leq(n / 2)\right\}$.

Lemma 1. Let $W$ be a locating-dominating set of $G$. Then, for $1 \leq i \leq(n / 2), x_{i} \in W$ or $y_{i} \in W$.

Proof. Suppose that there exists $i \in\{1,2, \ldots,(n / 2)\}$ such that $x_{i}, y_{i} \notin W$. Note that every vertex in $V(G)\left\{x_{i}, y_{i}\right\}$ is adjacent to both $x_{i}$ and $y_{i}$. So, we will obtain that $N_{G}\left(x_{i}\right) \cap W=N_{G}\left(y_{i}\right) \cap W$, a contradiction.

Lemma 2. A vertex set $\left\{x_{i} \mid 1 \leq i \leq(n / 2)\right\}$ is a locatingdominating set of $G$.

Proof. Let $S=\left\{x_{i} \mid 1 \leq i \leq(n / 2)\right\}$. Then, $V(G) \backslash S=\left\{y_{i} \mid 1 \leq i\right.$ $\leq(n / 2)\}$. Let us consider $y_{i}$ and $y_{j}$ for $1 \leq i<j \leq(n / 2)$. Since $x_{i} \in N_{G}\left(y_{j}\right)$ but $x_{i} \notin N_{G}\left(y_{i}\right)$, we obtain $\varnothing \neq N_{G}\left(y_{i}\right) \cap$ $S \neq N_{G}\left(y_{j}\right) \cap S \neq \varnothing$. Therefore, $S$ is a locating-dominating set of $G$.

\section{3. $(n-3)$-Regular Graph and Proof of Theorem 2}

In this section, we define $G$ as an (n-3)-regular graph of order $n \geq 5$. Note that $G$ contains a subgraph which is isomorphic to $K_{m} \backslash E\left(C_{m}\right)$ where $m \in\{3,4, \ldots, n\}$. Let $G^{\prime} \subseteq G$ and $G^{\prime}=K_{m} \backslash E\left(C_{m}\right)$ where $m \in\{3,4, \ldots, n\}$. Then, every vertex in $G^{\prime}$ is adjacent to all vertices of $G \backslash G^{\prime}$. In Lemma 3, we show that every subgraph is contributed to a locating-dominating set of $G$, which is a direct consequence of Observation 1 which has been proved by Henning and Oellermann [6].

Observation 1 (see [6]). Let $W$ be a locating-dominating set of a connected graph $G$. If there exists two distinct vertices $u, v \in V(G)$ such that $d_{G}(u, z)=d_{G}(v, z)$ for all $z \in V(G) \backslash$ $\{u, v\}$, then $u \in W$ or $v \in W$.

Lemma 3. For $n \geq 5$, let $G^{\prime} \subseteq G$ and $G^{\prime}=K_{m} \backslash E\left(C_{m}\right)$ where $m \in\{3,4, \ldots, n\}$. If $W$ is a locating-dominating set of $G$, then $W \cap V\left(G^{\prime}\right) \neq \varnothing$.

From Lemma 3, we have Lemma 4.

Lemma 4. For $n \geq 5$, let $G^{\prime} \subseteq G$ and $G^{\prime}=K_{m} \backslash E\left(C_{m}\right)$ where $m \in\{3,4, \ldots, n\}$. Let $W$ be a locating-dominating set of $G$ and $W^{\prime}=V\left(G^{\prime}\right) \cap W$. Then, every two distinct vertices $u, v \in V\left(G^{\prime}\right) \backslash W^{\prime}$ satisfies $N_{G^{\prime}}(u) \cap W^{\prime} \neq N_{G^{\prime}}(v) \cap W^{\prime}$. 
Proof. Suppose that there exists two distinct vertices $u, v \in V\left(G^{\prime}\right) \backslash W^{\prime}$ such that $N_{G^{\prime}}(u) \cap W^{\prime}=N_{G^{\prime}}(v) \cap W^{\prime}$. Note that $N_{G^{\prime}}(u) \cap W^{\prime}=N_{G}(u) \cap W^{\prime}$. Let $G^{*}=G \backslash G^{\prime}$ and $W^{*}=W \backslash W^{\prime}$. Since $N_{G}(u) \cap V\left(G^{*}\right)=V\left(G^{*}\right)=N_{G}(v) \cap$ $V\left(G^{*}\right)$, we obtain $N_{G}(u) \cap W=\left(N_{G}(u) \cap W^{*}\right) \cup\left(N_{G}\right.$ $\left.(u) \cap W^{\prime}\right)=\left(N_{G}(v) \cap W^{*}\right) \cup\left(N_{G}(v) \cap W^{\prime}\right)=N_{G}(v) \cap W$, a contradiction.

By Lemma 4, if $W$ is a locating-dominating set of $G$, then we can say that $V\left(G^{\prime}\right) \cap W$ is a locating-dominating set of a subgraph $G^{\prime}$ of $G$. Note that, in this case, for $u \in V\left(G^{\prime}\right)$, $N_{G^{\prime}}(u) \cap V\left(G^{\prime}\right) \cap W$ can be an empty set. In Lemma 5 , we provide a locating-dominating set of a subgraph $G^{\prime}=K_{m} \backslash E\left(C_{m}\right)$ of $G$ where $m \in\{3,4,5,6\}$.

Lemma 5. For $n \geq 5$, let $G^{\prime} \subseteq G$ and $G^{\prime}=K_{m} \backslash E\left(C_{m}\right)$ where $m \in\{3,4,5,6\}$. Let $W$ be a locating-dominating set of $G$. Then,

$$
\left|W \cap V\left(G^{\prime}\right)\right| \geq \begin{cases}3, & \text { if } m=n=6 \\ 2, & \text { otherwise. }\end{cases}
$$

The lower bounds are sharp.

Proof. Let $V\left(G^{\prime}\right)=\left\{x_{1}, x_{2}, \ldots, x_{m}\right\}$ and $E\left(C_{m}\right)=$ $\left\{x_{1} x_{2}, x_{2} x_{3}, \ldots, x_{m-1} x_{m}, x_{m} x_{1}\right\}$ where $m \in\{3,4,5,6\}$. We distinguish two cases.

(1) $\left|W \cap V\left(G^{\prime}\right)\right| \geq 3$ for $m=n=6$.

In this case, $G^{\prime}=G$. Suppose that $\left|W \cap V\left(G^{\prime}\right)\right| \leq 2$. Let $W^{\prime}$ be a locating-dominating set of $G^{\prime}$. Then, there exists $u, v \in V\left(G^{\prime}\right) \backslash W^{\prime}$ such that either $u \neq v$ and $\quad N_{G^{\prime}}(u) \cap W^{\prime}=N_{G^{\prime}}(v) \cap W^{\prime}, \quad$ or $N_{G^{\prime}}(u) \cap W^{\prime}=\varnothing$, a contradiction.

Now, we define $S=\left\{x_{1}, x_{3}, x_{5}\right\}$. Since $N_{G^{\prime}}\left(x_{2}\right)$ $\cap S=\left\{x_{5}\right\}, \quad N_{G^{\prime}}\left(x_{4}\right) \cap S=\left\{x_{1}\right\}, \quad$ and $N_{G^{\prime}}\left(x_{6}\right) \cap$ $S=\left\{x_{3}\right\}$, the vertex set $S$ is a locating-dominating set of $G^{\prime}$.

(2) $\left|W \cap V\left(G^{\prime}\right)\right| \geq 2$ for $m \neq 6$ or $n \neq 6$.

Suppose that $\left|W \cap V\left(G^{\prime}\right)\right| \leq 1$. If $\left|W \cap V\left(G^{\prime}\right)\right|=0$, then for two distinct vertices $u, v \in V\left(G^{\prime}\right) \backslash W$, $N_{G}(u) \cap W=W=N_{G}(v) \cap W$, a contradiction. So, we assume that $\left|W \cap V\left(G^{\prime}\right)\right|=1$. Let $W \cap V\left(G^{\prime}\right)=\left\{x_{i}\right\}$ with $i \in\{1,2, \ldots, m\}$. Let $x_{j}$ and $x_{k}$ be two different vertices in $G^{\prime}$ such that $x_{j} x_{i}, x_{k} x_{i} \notin E\left(G^{\prime}\right)$. So, $N_{G^{\prime}}\left(x_{j}\right) \cap W=\varnothing=N_{G^{\prime}}$ $\left(x_{k}\right) \cap W$. Since every vertex in $G \backslash G^{\prime}$ is adjacent to both $x_{j}$ and $x_{k}$, we have $N_{G}\left(x_{j}\right) \cap W=$ $W=N_{G}\left(x_{k}\right) \cap W$, a contradiction.

For the sharpness, we define a vertex set $S_{m}$ as follows:

$$
S_{m}= \begin{cases}\left\{x_{1}, x_{2}\right\}, & \text { if } m \in\{3,4,5\}, \\ \left\{x_{1}, x_{3}\right\}, & \text { if } m=6\end{cases}
$$

We will show that $S_{m}$ is a locating-dominating set of a subgraph $G^{\prime}$ of $G$. Let us consider vertices in $V\left(G^{\prime}\right) \backslash S_{m}$. (a) $m=3$ : we obtain that $\left|V\left(G^{\prime}\right) \backslash S_{m}\right|=1$. So, it is clear that $S_{m}$ is a locating-dominating set of $G^{\prime}$.

(b) $m=4$ : we obtain that $V\left(G^{\prime}\right) \backslash S_{m}=\left\{x_{3}, x_{4}\right\}$. Note that $N_{G^{\prime}}\left(x_{3}\right) \cap S_{m}=\left\{x_{1}\right\} \neq\left\{x_{2}\right\}=N_{G^{\prime}}\left(x_{4}\right) \cap S_{m}$. Therefore, $S_{m}$ is a locating-dominating set of $G^{\prime}$.

(c) $m=5$ : we obtain that $V\left(G^{\prime}\right) \backslash S_{m}=\left\{x_{3}, x_{4}, x_{5}\right\}$. It is easy to see that $N_{G^{\prime}}\left(x_{3}\right) \cap S_{m}=\left\{x_{1}\right\}, N_{G^{\prime}}\left(x_{4}\right)$ $\cap S_{m}=\left\{x_{1}, x_{2}\right\}$, and $N_{G^{\prime}}\left(x_{5}\right) \cap S_{m}=\left\{x_{2}\right\}$. Therefore, it is clear that $S_{m}$ is a locating-dominating set of $G^{\prime}$.

(d) $m=6$ : we obtain that $V\left(G^{\prime}\right) \backslash S_{m}=\left\{x_{2}, x_{4}, x_{5}, x_{6}\right\}$. It is easy to see that $N_{G^{\prime}}\left(x_{2}\right) \cap S_{m}=\varnothing, N_{G^{\prime}}\left(x_{4}\right) \cap$ $S_{m}=\left\{x_{1}\right\}, N_{G^{\prime}}\left(x_{5}\right) \cap S_{m}=\left\{x_{1}, x_{3}\right\}$, and $N_{G^{\prime}}\left(x_{6}\right) \cap$ $S_{m}=\left\{x_{3}\right\}$. Therefore, it is clear that $S_{m}$ is a locatingdominating set of $G^{\prime}$.

Remark 1 . We can say that the locating-dominating number of $G^{\prime}$ in Lemma 5 is given by

$$
\lambda\left(G^{\prime}\right)= \begin{cases}2, & \text { if } m \in\{3,4,5,6\} \text { and } n>m, \\ 3, & \text { if } n=m=6 .\end{cases}
$$

Now, let us consider $G^{\prime} \subseteq G$ and $G^{\prime}=K_{m} \backslash E\left(C_{m}\right)$ for $m \geq 7$. Thus, the order of $G$ must be $n \geq 7$. For $n \geq 7$, in order to determine a locating-dominating set of $G^{\prime}=K_{m} \backslash E\left(C_{m}\right)$ for $7 \leq m \leq n$, we define some definitions. For $u, v \in V(G)$, let $P(u, v)$ be a shortest $(u, v)$-path in $C_{m}$. So, all edges in $P(u, v)$ are not element of $E\left(G^{\prime}\right)$. Let $W \subseteq V\left(G^{\prime}\right)$. For $u, v \in W$, let us consider the set of vertices of $P(u, v) \backslash\{u, v\}$. If all vertices of $P(u, v) \backslash\{u, v\}$ are not in $W$, then the set of vertices in $P(u, v) \backslash\{u, v\}$ is called a gap between $u$ and $v$. Then, we called vertices $u$ and $v$ as the end points of gap. The two gaps are called neighbouring gaps if they have common end point. These definitions were firstly introduced by Buczkowski et al. [18]. They used this gap technique to determine the metric dimension of wheel graphs. In lemma 6 , we provide the necessary and sufficient conditions for a locating-dominating set of $G^{\prime}$ which is related to gap definition.

Lemma 6. For $n \geq 7$, let $G^{\prime}=K_{m} \backslash E\left(C_{m}\right)$ where $m \in\{7,8$, $\ldots, n\}$. The vertex set $W \subseteq V\left(G^{\prime}\right)$ is a locating-dominating set of $G^{\prime}$ if and only if $W$ satisfies all conditions as follows:

(1) Every gap with respect to $W$ contains at most 3 vertices

(2) $W$ contains at most one gap of 3 vertices

(3) If $A$ is a gap with respect to $W$, containing 2 or 3 vertices, then any neighbouring gaps of $A$ have at most one vertex

Proof. $\quad \Rightarrow$ We will prove all three conditions by contradiction.

(1) Suppose that there exists a gap with respect to $W$ containing at least 4 vertices. 
Let $a_{1}, a_{2}, a_{3}, a_{4} \in V\left(G^{\prime}\right)$ where $a_{i} a_{i+1} \in E\left(C_{m}\right)$ with $1 \leq i \leq 3$ and all those vertices are not in $W$. Then, we have $\quad N_{G^{\prime}}\left(a_{2}\right) \cap W=W=N_{G^{\prime}}\left(a_{3}\right) \cap W, \quad$ a contradiction.

(2) Suppose that there exists two gaps containing 3 vertices.

Let $\left\{a_{1}, a_{2}, a_{3}\right\}$ and $\left\{b_{1}, b_{2}, b_{3}\right\}$ be two different gaps where $a_{i} a_{i+1}, b_{i} b_{i+1} \in E\left(C_{m}\right)$ with $1 \leq i \leq 2$. Then, we have $\quad N_{G^{\prime}}\left(a_{2}\right) \cap W=W=N_{G^{\prime}}\left(b_{2}\right) \cap W, \quad$ a contradiction.

(3) Suppose that there exists a neighbouring gap of $A$ containing at least 2 vertices.

Let $a_{1}, a_{2}, a_{3}, a_{4}, a_{5} \in V\left(G^{\prime}\right)$ where $a_{i} a_{i+1} \in E\left(C_{m}\right)$ with $1 \leq i \leq 4$ and $a_{3}$ is the only element of $W$ among them. Then, we have $N_{G^{\prime}}\left(a_{2}\right) \cap W=W \backslash\left\{a_{3}\right\}$ $=N_{G^{\prime}}\left(a_{4}\right) \cap W$, a contradiction.

$(\Leftarrow)$ Let $S \subseteq V\left(G^{\prime}\right)$ satisfying all three conditions above. Since $m \geq 7$, we obtain $|S| \geq 3$. Now, we consider a vertex $u \in V\left(G^{\prime}\right) \backslash S$.

(i) $u$ belongs to a gap containing one vertex.

Let $a$ and $b$ be two end points of this gap. So, $u$ is the only vertex which is not adjacent to $a$ and $b$. Since $|S| \geq 3$ and for every vertex $x \in V\left(G^{\prime}\right) \backslash(S \cup\{u\})$, $a \in N_{G^{\prime}}(x)$ or $b \in N_{G^{\prime}}(x)$, we obtain $\varnothing \neq N_{G^{\prime}}$ $(u) \cap S \neq N_{G^{\prime}}(x) \cap S \neq \varnothing$.

(ii) $u$ belongs to a gap containing two vertices.

Let $a$ and $b$ be two end points of this gap. Without loss of generality, let $a u \in E\left(C_{m}\right)$. So, $N_{G^{\prime}}(u)$ $\cap S=S \backslash\{a\}$. For $x \in V\left(G^{\prime}\right) \backslash(S \cup\{u)\}$, if $a \in N_{G^{\prime}}(x)$, then $\varnothing \neq N_{G^{\prime}}(u) \cap S \neq N_{G^{\prime}}(x) \cap S \neq \varnothing$; otherwise, $x$ belongs to a gap containing one vertex. From (i) above, we obtain that $\varnothing \neq N_{G^{\prime}}(u) \cap S \neq N_{G^{\prime}}$ $(x) \cap S \neq \varnothing$.

(iii) $u$ belongs to a gap containing three vertices.

Let $c_{1}, c_{2}$, and $c_{3}$ be a gap of three vertices where $c_{i} c_{i+1} \in E\left(C_{m}\right)$ with $1 \leq i \leq 2$ and $a$ and $b$ be end points of this gap. Let $a c_{1}, b c_{3} \in E\left(C_{m}\right)$. If $u=c_{2}$, then $N_{G^{\prime}}(u) \cap S=S$. Since $u$ is the only vertex having this property, we obtain that for every vertex $x \in V\left(G^{\prime}\right) \backslash(S \cup\{u)\} \quad$ and $\quad \varnothing \neq N_{G^{\prime}}(u) \cap S \neq N_{G^{\prime}}$ $(x) \cap S \neq \varnothing$. If $u=c_{1}$, then $N_{G^{\prime}}(u) \cap S=S \backslash\{a\}$. For $x \in V\left(G^{\prime}\right) \backslash(S \cup\{u)\}$, if $a \in N_{G^{\prime}}(x)$, then $\varnothing \neq$ $N_{G^{\prime}}(u) \cap S \neq N_{G^{\prime}}(x) \cap S \neq \varnothing$; otherwise, $x$ belongs to a gap containing one vertex. From (i) above, we obtain that $\varnothing \neq N_{G^{\prime}}(u) \cap S \neq N_{G^{\prime}}(x) \cap S \neq \varnothing$.

Now, we are ready to prove Theorem 2 .

Proof of Theorem 2 .

For $m \geq 7$, let $H=K_{m} \backslash E\left(C_{m}\right)$ where $V(H)=\left\{x_{1}\right.$, $\left.x_{2}, \ldots, x_{m}\right\}$ and $E\left(C_{m}\right)=\left\{x_{1} x_{2}, x_{2} x_{3}, \ldots, x_{m-1} x_{m}, x_{m} x_{1}\right\}$. We distinguish two cases as follows:

(1) $\lambda(H) \leq\lceil(2 m-2) / 5\rceil$ : we distinguish five cases of $m$ as follows: (a) $m \equiv 0(\bmod 5)$ : let $m=5 k$ for an integer $k \geq 2$. Then, $\lceil(2 m-2) / 5\rceil=\lceil(10 k-2) / 5\rceil=2 k$. We define $W=\left\{x_{2}, x_{6}\right\} \cup\left\{x_{5 i+3}, x_{5 i+6} \mid \quad 1 \leq i \leq\right.$ $k-2\} \cup\left\{x_{5 k-2}, x_{5 k}\right\}$. Since $|W|=2 k$ and satisfies all conditions in Lemma 6 , then $W$ is a locating-dominating set of $H$.

(b) $m \equiv 1(\bmod 5):$ let $m=5 k+1$ for an integer $k \geq 2$. Then, $\lceil(2 m-2) / 5\rceil=\lceil 10 k / 5\rceil=2 k$. We define $W=\left\{x_{2}, x_{6}\right\} \cup\left\{x_{5 i+3}, x_{5 i+6} \mid \quad 1 \leq i \leq\right.$ $k-2\} \cup\left\{x_{5 k-2}, x_{5 k+1}\right\}$. Since $|W|=2 k$ and satisfies all conditions in Lemma 6, then $W$ is a locating-dominating set of $H$.

(c) $m \equiv 2(\bmod 5):$ let $m=5 k+2$ for an integer $k \geq 1$. Then, $\lceil(2 m-2) / 5\rceil=\lceil(10 k+2) / 5\rceil$ $=2 k+1$. We define $W=\left\{x_{2}, x_{6}\right\} \cup\left\{x_{5 i+3}\right.$, $\left.x_{5 i+6} \mid 1 \leq i \leq k-1\right\} \cup\left\{x_{5 k+2}\right\}$. Since $|W|=2 k+$ 1 and satisfies all conditions in Lemma 6 , then $W$ is a locating-dominating set of $H$.

(d) $m \equiv 3(\bmod 5):$ let $m=5 k+3$ for an integer $k \geq 1$. Then, $\lceil(2 m-2) / 5\rceil=\lceil(10 k+4) / 5\rceil=2$ $k+1$. We define $W=\left\{x_{2}, x_{6}\right\} \cup\left\{x_{5 i+3}, x_{5 i+6} \mid\right.$ $1 \leq i \leq k-1\} \cup\left\{x_{5 k+3}\right\}$. Since $|W|=2 k+1$ and satisfies all conditions in Lemma 6, then $W$ is a locating-dominating set of $H$.

(e) $m \equiv 4(\bmod 5):$ let $m=5 k+4$ for an integer $k \geq 1$. Then, $\lceil(2 m-2) / 5\rceil=\lceil(10 k+6) / 5\rceil=$ $2 k+2$. We define $W=\left\{x_{2}, x_{6}\right\} \cup\left\{x_{5 i+3}\right.$, $\left.x_{5 i+6} \mid 1 \leq i \leq k-1\right\} \cup\left\{x_{5 k+3}, x_{5 k+4}\right\}$. Since $|W|=2 k+2$ and satisfies all conditions in Lemma 6 , then $W$ is a locating-dominating set of $H$.

(2) $\lambda(H) \geq\lceil(2 m-2) / 5\rceil$ : let $S$ be a locating-dominating set of $H$ with minimum cardinality. We consider two following cases:

(a) $|S|$ is even.

Let $|S|=2 k$ for a positive integer $k$. So, the number of gap of $H$ with respect to $S$ is $2 k$. Since $S$ must be satisfy all conditions in Lemma 6 , the number of gap containing at least 2 vertices is at most $k$. It follows that the number of vertex which is not in $S$ is at most $3 k+1$. So, we obtain that $k \geq((m-1) / 5)$. Therefore,

$$
\begin{aligned}
\lambda(H) & =|S|=2 k \geq 2 \cdot\left\lceil\frac{m-1}{5}\right\rceil \\
& =\left\lceil\frac{2 m-2}{5}\right\rceil .
\end{aligned}
$$

(b) $|S|$ is odd.

Let $|S|=2 k+1$ for a positive integer $k$. So, the number of gap of $H$ with respect to $S$ is $2 k+1$. Since $S$ must be satisfy all conditions in Lemma 6 , the number of gap containing at least 2 vertices is at most $k$. It follows that the number of vertex which is not in $S$ is at most $3 k+2$. So, we obtain that $k \geq((m-3) / 5)$. Therefore, 


$$
\begin{aligned}
\lambda(H) & =|S|=2 k+1 \geq 2 \cdot\left\lceil\frac{m-3}{5}\right\rceil+1 \\
& =\left\lceil\frac{2 m-1}{5}\right\rceil \geq\left\lceil\frac{2 m-2}{5}\right\rceil .
\end{aligned}
$$

\section{4. $(n-3)$-Regular Graph and Proof of Theorem 3}

For $n \geq 5$, we consider certain cycles contained in a complete graph $K_{n}$. For $1 \leq r \leq\lfloor n / 3\rfloor$, let $R_{1}, R_{2}, \ldots, R_{r}$ be $r$ disjoint cycles contained in $K_{n}$ such that $V\left(R_{1}\right) \cup V\left(R_{2}\right) \cup \cdots \cup V$ $\left(R_{r}\right)=V\left(K_{n}\right)$. Note that an $(n-3)$-regular graph is isomorphic to $K_{n} \backslash\left(E\left(R_{1}\right) \cup E\left(R_{2}\right) \cup \cdots \cup E\left(R_{r}\right)\right)$. In case $r=1$, the locating-dominating number of an $(n-3)$-regular graph of order at least 7 has been determined in Theorem 2 . Now, we will determine the locating-dominating number of an $(n-3)$-regular graph of order $n \geq 5$ with $1 \leq r \leq\lfloor n / 3\rfloor$.

Let $\quad G=K_{n} \backslash\left(E\left(R_{1}\right) \cup E\left(R_{2}\right) \cup \cdots \cup E\left(R_{r}\right)\right) \quad$ and $m_{i}=\left|V\left(R_{i}\right)\right|$ where $1 \leq i \leq r$. Let $G_{i}$ be a subgraph of $G$ where $G_{i}=K_{m_{i}} \backslash E\left(R_{i}\right)$. Considering Lemma 4, a locating-dominating set of $G$ consists of a locating-dominating set of $G_{i}$ with $1 \leq i \leq r$. Therefore, we obtain that

$$
\lambda(G) \geq \lambda\left(G_{1}\right)+\lambda\left(G_{2}\right)+\cdots+\lambda\left(G_{r}\right) .
$$

Note that a locating-dominating of $G$ also must satisfy all three conditions in Lemma 6. Let $W=\cup_{i=1}^{r} W_{i}$ where $W_{i}$ is a locating-dominating set of $G_{i}$ where $\left|W_{i}\right|=\lambda\left(G_{i}\right)$. If there exists distinct $i, j \in\{1,2, \ldots, r\}$ such that both locatingdominating sets $G_{i}$ and $G_{j}$ contain a gap of three vertices, then we must add at least one more vertex on $W$. So, we need to know the gap properties of a locating-dominating set of $G_{i}$.

Lemma 7. For $n \geq 5$, let $G$ be an $(n-3)$-regular graph. Let $G^{\prime} \subseteq G$ such that $G^{\prime}=K_{m} \backslash E\left(C_{m}\right)$ with $m \in\{3,4, \ldots, n\}$.

(1) If $m=3, n>m=5$, or $m \equiv 0,2,4(\bmod 5)$, then there exists a locating-dominating set of $G^{\prime}$ where every gap contains at most two vertices.

(2) If $m=n=5$ or $m \equiv 1,3(\bmod 5)$ with $m \neq 3$, then $a$ locating-dominating set of $G^{\prime}$ has a gap containing three vertices.

Proof. First, let $m=3, n>m=5$, or $m \equiv 0,2,4(\bmod 5)$. Note that, in this case of $m$, we have $G^{\prime} \subset G$. Let $V\left(G^{\prime}\right)=$ $\left\{x_{1}, x_{2}, \ldots, x_{m}\right\}$ and $E\left(C_{m}\right)=\left\{x_{1} x_{2}, x_{2} x_{3}, \ldots, x_{m-1} x_{m}\right.$, $\left.x_{m} x_{1}\right\}$. We distinguish five cases as follows:

(1) $m=3$ or $m=5$ : by Remark $1, \lambda\left(G^{\prime}\right)=2$. We define $W^{\prime}=\left\{x_{1}, x_{3}\right\}$.

(2) $m=4$ : by Remark $1, \lambda\left(G^{\prime}\right)=2$. We define $W^{\prime}$ $=\left\{x_{1}, x_{4}\right\}$.

(3) $m \equiv 0(\bmod 5)$ : let $m=5 k$ for integer $k \geq 2$. By Theorem 2, $\lambda\left(G^{\prime}\right)=2 k$. We define $W^{\prime}=\left\{x_{1}, x_{4}\right.$, $\left.x_{6}, x_{9}\right\} \cup\left\{x_{5 i+6}, x_{5 i+9} \mid 1 \leq i \leq k-2\right\}$.

(4) $m \equiv 2(\bmod 5):$ let $m=5 k+2$ for integer $k \geq 1$. By Theorem 2, $\lambda\left(G^{\prime}\right)=2 k+1$. We define $W^{\prime}=\left\{x_{1}\right.$, $\left.x_{4}, x_{6}\right\} \cup\left\{x_{5 i+4}, x_{5 i+6} \mid 1 \leq i \leq k-1\right\}$.
(5) $m \neq 4$ and $m \equiv 4(\bmod 5)$ : let $m=5 k+4$ for integer $k \geq 1$. By Theorem $2, \lambda\left(G^{\prime}\right)=2 k+2$. We define $W^{\prime}=\left\{x_{1}, x_{4}, x_{6}, x_{9}\right\} \cup\left\{x_{5 i+6}, x_{5 i+9} \mid 1 \leq i \leq k-1\right\}$.

Note that every gap with respect to $W^{\prime}$ above contains at most two vertices. Since $W^{\prime}$ satisfies Lemma 6 , then $W^{\prime}$ is a locating-dominating set of $G^{\prime}$.

Now, let $m=n=5$ or $m \equiv 1,3(\bmod 5)$ with $m \neq 3$. Suppose that every gap in $G^{\prime}$ contains at most two vertices. We distinguish three cases as follows:

(1) $m \equiv 1(\bmod 5):$ let $m=5 k+1$ for integer $k \geq 1$. By Theorem 2, $\lambda\left(G^{\prime}\right)=2 k$. Let $W$ be a locatingdominating set of $G^{\prime}$ with $2 k$ vertices. By Lemma 6 , $k$ gaps with respect to $W$, containing one vertex, and $k$ other gaps with respect to $W$, containing two vertices. Thus, the total number of vertices of $G^{\prime}$ which are not element of $W$ is $3 k$. It follows that $m=\left|V\left(G^{\prime}\right) \backslash W\right|+|W|=3 k+2 k=5 k$, contradiction.

(2) $m \neq 3$ and $m \equiv 3(\bmod 5)$ : let $m=5 k+3$ for integer $k \geq 1$. By Theorem $2, \lambda\left(G^{\prime}\right)=2 k+1$. Let $W$ be a locating-dominating set of $G^{\prime}$ with $2 k+1$ vertices. By Lemma 6, $k+1$ gaps with respect to $W$, containing one vertex, and $k$ other gaps with respect to $W$, containing two vertices. Thus, the total number of vertices of $G^{\prime}$ which are not element of $W$ is $3 k+1$. It follows that $m=\left|V\left(G^{\prime}\right) \backslash W\right|+|W|=$ $(3 k+1)+2 k=5 k+1$, a contradiction.

(3) $m=n=5$ : thus, $G=G^{\prime}$. Let $V\left(G^{\prime}\right)=\left\{x_{1}, x_{2}, \ldots\right.$, $\left.x_{5}\right\}$ and $E\left(C_{5}\right)=\left\{x_{1} x_{2}, x_{2} x_{3}, \ldots, x_{4} x_{5}, x_{5} x_{1}\right\}$. By Remark $1, \lambda\left(G^{\prime}\right)=2$. Since every gap in $G^{\prime}$ contains at most two vertices, without loss of generality, let $W$ be a locating-dominating set of $G^{\prime}$ where $W=\left\{x_{1}, x_{3}\right\}$. Since $N_{G}\left(x_{2}\right)=\left\{x_{4}, x_{5}\right\}$, we obtain that $N_{G}\left(x_{2}\right) \cap W=\varnothing$, a contradiction.

Now, we are ready to prove Theorem 3.

Proof of Theorem 3.

The first case for $\lambda(G)$ is a direct consequence of Theorem 2, Lemmas 6 and 7, and Remark 1.

For the last case, let $H_{m_{1}}, H_{m_{2}}, \ldots, H_{m_{k}}$ be disjoint $k$ subgraphs of $G$ such that $H_{m_{i}}=K_{m_{i}} \backslash E\left(C_{m_{i}}\right)$ where $1 \leq i \leq k$, $m_{i} \neq 3$, and $m_{i} \equiv 1$ or $3(\bmod 5)$. Let $B_{i}$ be a locating-dominating set of $H_{m_{i}}$ with $\lambda\left(H_{m_{i}}\right)$ vertices. By Lemma $7, B_{i}$ has a gap containing three vertices, say $a_{1}^{i}, a_{2}^{i}$, and $a_{3}^{i}$ where $a_{j}^{i} a_{j+1}^{i} \notin E\left(H_{m_{i}}\right)$ with $1 \leq j \leq 2$. We define $B_{i}^{\prime}=B_{i} \cup\left\{a_{2}^{i}\right\}$. It is easy to see that $B_{i}^{\prime}$ is a locating-dominating set of $H_{m_{i}}$ which all the gaps contain at most two vertices. So, by using this property to subgraphs $H_{m_{1}}, H_{m_{2}}, \ldots, H_{m_{k-1}}$ of $G$, Theorem 2, Lemmas 6 and 7, and Remark 1, we prove the last case.

\section{Data Availability}

This research article is the theoretical study of locatingdominating set in graphs. There is no data supporting used. All results in the manuscript can be obtained by the analytical method. 


\section{Conflicts of Interest}

The authors declare there are no conflicts of interest.

\section{Authors' Contributions}

A.K.A.G and S.W.S conceptualized and wrote the study. S.W.S revised the study. All authors have read and agreed to the published version of the manuscript.

\section{Acknowledgments}

This research was partially supported by Riset Unggulan of Bandung Institute of Technology 2021 Indonesia.

\section{References}

[1] P. J. Slater, "Domination and location in acyclic graphs," Networks, vol. 17, no. 1, pp. 55-64, 1987.

[2] P. J. Slater, "Dominating and reference sets in a graph," Journal of Mathematical and Physical Sciences, vol. 22, pp. $445-455,1988$.

[3] I. Charon, O. Hudry, and A. Lobstein, "Minimizing the size of an identifying or locating-dominating code in a graph is NPhard," Theoretical Computer Science, vol. 290, no. 3, pp. 2109-2120, 2003.

[4] N. Bertrand, I. Charon, O. Hudry, and A. Lobstein, "Identifying and locating-dominating codes on chains and cycles," European Journal of Combinatorics, vol. 25, no. 7, pp. 969987, 2004.

[5] G. R. Argiroffo, S. M. Bianchi, and A. K. Wagler, "A polyhedral approach to locating-dominating sets in graphs," Electronic Notes in Discrete Mathematics, vol. 50, pp. 89-94, 2015.

[6] M. A. Henning and O. R. Oellermann, "Metric-locatingdominating sets in graphs," Ars Combinatoria, vol. 73, pp. 129-141, 2004.

[7] J. Cáceres, C. Hernando, M. Mora, I. M. Pelayo, and M. L. Puertas, "Locating-dominating codes: bounds and extremal cardinalities," Applied Mathematics and Computation, vol. 220, pp. 38-45, 2013.

[8] C. Hernando, M. Mora, and I. M. Pelayo, "Locating domination in bipartite graphs and their complements," Discrete Applied Mathematics, vol. 263, pp. 195-203, 2019.

[9] F. Foucaud, M. A. Henning, and C. Löwenstein, "Locatingdominating sets in twin-free graphs," Discrete Applied Mathematics, vol. 200, pp. 52-58, 2016.

[10] D. Garijo, A. González, and A. Márquez, “The difference between the metric dimension and the determining number of a graph," Applied Mathematics and Computation, vol. 249, pp. 487-501, 2014.

[11] M. Fazil, I. Javaid, M. Salman, and U. Ali, "Locating-dominating sets in hypergraphs," Periodica Mathematica Hungarica, vol. 72, no. 2, pp. 224-234, 2016.

[12] C. Balbuena, F. Foucaud, and A. Hansberg, "Locating-dominating sets and identifying codes in graphs of girth at least 5," Electronic Journal of Combinatorics, vol. 22, no. 2, 2015.

[13] G. Rajasekar and K. Nagarajan, "Algorithm for finding location domination number of a graph connected by a bridge," International Journal of Pure and Applied Mathematics, vol. 118, no. 6, pp. 313-321, 2018.

[14] S. R. Canoy Jr, G. A. Malacas, and D. Tarepe, "Locating dominating sets in graphs," Applied Mathematical Sciences, vol. 8, no. 8, pp. 4381-4388, 2014.
[15] A. A. Pribadi and S. W. Saputro, "On locating-dominating number of comb product graphs," Indonesian Journal of Combinatorics, vol. 4, no. 1, pp. 27-33, 2020.

[16] M. Murtaza, M. Fazil, and I. Javaid, "Locating-dominating sets of functigraphs," Theoretical Computer Science, vol. 799, pp. 115-123, 2019.

[17] G. Argiroffo, S. Bianchi, Y. Lucarini, and A. Wagler, "The identifying code, the locating-dominating, the open locatingdominating and the locating total-dominating problems under some graph operations," Electronic Notes in Theoretical Computer Science, vol. 346, pp. 135-145, 2019.

[18] P. S. Buczkowski, G. Chartrand, C. Poisson, and P. Zhang, "On $k$-dimensional graphs and their bases," Periodica Mathematica Hungarica, vol. 46, no. 1, pp. 9-15, 2003. 